\title{
Critical Links and Nonlocal Rerouting in Complex Supply Networks
}

\author{
Dirk Witthaut, ${ }^{1,2,3}$ Martin Rohden, ${ }^{1,4,5}$ Xiaozhu Zhang, ${ }^{1}$ Sarah Hallerberg, ${ }^{1}$ and Marc Timme ${ }^{1,6}$ \\ ${ }^{1}$ Network Dynamics, Max Planck Institute for Dynamics and Self-Organization (MPIDS), 37077 Göttingen, Germany \\ ${ }^{2}$ Forschungszentrum Jülich, Institute for Energy and Climate Research-Systems Analysis \\ and Technology Evaluation (IEK-STE), 52428 Jülich, Germany \\ ${ }^{3}$ Institute for Theoretical Physics, University of Cologne, 50937 Köln, Germany \\ ${ }^{4}$ IIIrd Institute of Physics, Faculty of Physics, Georg August University, 37077 Göttingen, Germany \\ ${ }^{5}$ Department of Physics and Earth Sciences, Jacobs University, 28759 Bremen, Germany \\ ${ }^{6}$ Institute for Nonlinear Dynamics, Faculty of Physics, Georg August University Göttingen, 37077 Göttingen, Germany
}

(Received 28 October 2015; published 30 March 2016)

\begin{abstract}
Link failures repeatedly induce large-scale outages in power grids and other supply networks. Yet, it is still not well understood which links are particularly prone to inducing such outages. Here we analyze how the nature and location of each link impact the network's capability to maintain a stable supply. We propose two criteria to identify critical links on the basis of the topology and the load distribution of the network prior to link failure. They are determined via a link's redundant capacity and a renormalized linear response theory we derive. These criteria outperform the critical link prediction based on local measures such as loads. The results not only further our understanding of the physics of supply networks in general. As both criteria are available before any outage from the state of normal operation, they may also help real-time monitoring of grid operation, employing countermeasures and support network planning and design.
\end{abstract}

DOI: 10.1103/PhysRevLett.116.138701

The robust operation of physical distribution and supply networks is fundamental for economy, industry, and our daily life. For instance, a reliable supply of electric power fundamentally underlies the function of most of our technical infrastructure [1-6]. In periods of high loads, the breakdown of a single element of the grid, such as a transmission line, can cause a global cascade of failures implying large-scale outages with potentially catastrophic consequences [3,7-15]. Periods of extreme loads are expected to become more likely in future grids, as power from renewable sources, such as wind turbines, is often generated far away from the consumers (e.g., off shore) and moreover strongly fluctuating $[2,16-18]$. It is thus crucial to understand which factors limit the robustness of supply networks and, in particular, to identify those links that are indispensable for network operation; compare also Refs. [5,19-24]. Are there simple characteristics in the physics of complex supply networks to identify which of their links are critical?

Many approaches to identify critical transmission lines in power grids or critical links in other supply networks fundamentally rely, for instance, on large-scale numerical simulations of detailed models that, after a local breakdown, emulate the future dynamics of large parts of the network [19,25-27]. Here, we propose a complementary approach to predict $a$ priori which links are critical in the sense that their failure yields a larger-scale malfunction (outage) in the network. We identify two concepts that reveal, prior to any outage, how the network topology jointly with the load distribution influences which links are critical: The first relies on the link's redundant capacity which we quantify, the second originates from a renormalized linear response theory we derive. Based on these theoretical insights, we propose two network-based criteria to identify critical links. A statistical evaluation suggests that the new measures predict critical links much more reliably than standard loads or flows.

Loads, flows, and critical links. - To obtain insights into mechanisms underlying large-scale outages and to pin down how the structure of a network determines its vulnerability, we base our analysis on a dynamic model of ac power grids $[23,28-30]$. Simpler generic models of supply networks and more complex load flow models of engineering yield qualitatively the same results [31]. The model describes the dynamics of the voltage phase angles $\theta_{j}(t)$ of all units $j=1, \ldots, N$, while the voltage magnitudes are approximately constant on the relevant time scales. Denoting by $\phi_{j}(t)=\theta_{j}(t)-\Omega t$ the difference to the reference phase oscillating with the grid's cycle frequency $\Omega$, e.g., $\Omega=2 \pi \times 50 \mathrm{~Hz}$, the equations of motion read

$$
\frac{d^{2} \phi_{j}}{d t^{2}}=P_{j}-\alpha \frac{d \phi_{j}}{d t}+\sum_{i=1}^{N} K_{i j} \sin \left(\phi_{i}-\phi_{j}\right)
$$

where $P_{j}$ is the effective demand or supply (of a consumer or sink where $P_{j}<0$ or a producer or source where $P_{j}>0$, respectively) of unit $j, \alpha$ is a damping constant, and the link capacities $K_{j i}=K_{i j} \geq 0$ are proportional to the susceptance of the transmission line $(i, j)$. 
The power flow

$$
F_{i j}=K_{i j} \sin \left(\phi_{i}-\phi_{j}\right)
$$

from unit $i$ to $j$ is then determined via the units' phase difference such that the (relative) load is given by $L_{i j}=F_{i j} / K_{i j}=\sin \left(\phi_{i}-\phi_{j}\right)$. Stable stationary operation is then given by a synchronized ("phase-locked") solution with fixed phase differences $\phi_{i}-\phi_{j}$ [1,30,51-59].

How can we identify which links are critical? The load distribution of a given supply network in normal stationary operation may serve as a first hint. Because of the distributed nature of sources and sinks and the topology of the network, some links are much more loaded than others (Fig. 1). Intuitively, rerouting the load of a highly loaded link should be harder than that of a less loaded one $[60,61]$. It has been shown that attacks on highly loaded links on average have more severe consequences than random failures (see, e.g., $[9,12]$ ).

Interestingly, whether or not the failure of a particular link induces network desynchronization is often not predictable by local measures such as the load. Single link
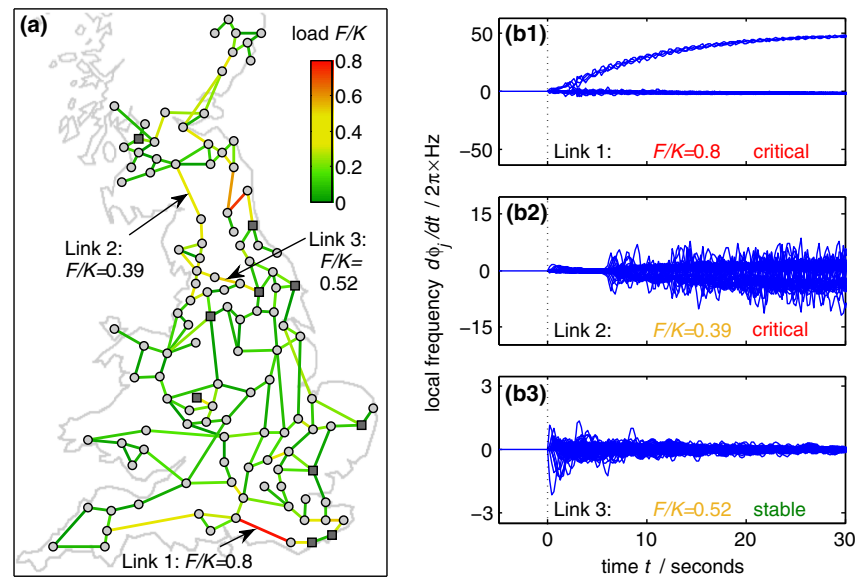

FIG. 1. Limits to predicting critical links by local measures. (a) Stationary loads in a coarse-grained model of the British power grid. For three links $(a, b)$ the loads $L_{a b}=F_{a b} / K_{a b}$ are indicated. (b) The load does not necessarily indicate whether a local failure causes a desynchronization and thus a major malfunction. (b1) A highly loaded link [labeled "link 1" in panel (a)] induces desynchronization and is thus critical. (b2) Moderately loaded link "2" still induces desynchronization and thus is also critical. (b3) In the same network, even a more heavily loaded link "3" does not yield desynchronization and leaves the network fully functional. Accordingly, link 3 is highly loaded but noncritical (stable). We analyze critical links for test networks based on the topology of the British transmission grid [10,28]: Transmission lines have a capacity $K_{0}=15 \mathrm{~s}^{-2}$, except for lines connecting to generator nodes which have doubled capacity. Ten nodes are randomly chosen as generators $\left(\square, P_{j}=+11 P_{0}\right)$ and the others as consumers $\left(\circ, P_{j}=-P_{0}\right)$, with $P_{0}=1 \mathrm{~s}^{-2}$, modeling a heavily loaded grid. Other test grids exhibit qualitatively the same phenomena [31]. failures may have completely distinct consequences for global network operation, largely independent of load: For instance, whereas the failure of one highly loaded link [link 1 in Fig. 1(a)] may cause a desynchronization of phases and thus a large-scale outage [see Fig. 1(b1)], the failure of a less and only moderately loaded link (link 2) may still induce large-scale outage [Fig. 1(b2)]. This notwithstanding, the failure of a third link (link 3) that is more highly loaded than link 2 is uncritical to network operation [Fig. 1(b3)]. So predicting outages based on a link's load alone may have substantial limitations.

In what follows, we classify all links of a network into "critical" ones, those whose failure induces long-term desynchronization and thus a nonfunctional network state, and "stable" ones, those whose failure leaves the network functional. In particular, we integrate the equations of motion (1) numerically starting from a stationary state of normal operation (phase locking). As it turns out, which links are critical depends jointly on the global topological structure of a network and its collective dynamics, in particular, on the link's location within the grid topology and the entire grid's load distribution.

Quantifying network redundancy.-For a supply network to remain stable, it needs sufficient options for rerouting the (directed) flow $F_{a b}$ originally assigned to a failing link $(a, b)$. Therefore, a sufficient degree of redundancy of the remaining network matters to reliably detect critical links. How much redundancy is sufficient?

To identify critical links, we quantify the redundancy as follows: If the link $(a, b)$ fails, the (directed) flow $F_{a b}$ has to reroute over alternative paths in the network. However, the links along these paths have only a limited residual capacity $K_{i j}-F_{i j}$ to take over this flow. We define the redundant capacity $K_{a b}^{\text {red }}$ of a link $(a, b)$ as the maximum flow that can be transmitted from unit $a$ to $b$ over the residual network excluding link $(a, b)$ (see [31] for an algorithm to determine it). The redundant capacity is approximately given by

$$
K_{a b}^{\mathrm{red}}=\sum_{\substack{\text { paths } \\ a \rightarrow b}} \min _{(i, j) \in a \rightarrow b}\left(K_{i j}-F_{i j}\right),
$$

that is, the minimum residual capacity across the links $(i, j)$ on a path $a \rightarrow b$, summed over different alternative paths $a \rightarrow b$ in the residual network which do not share a "bottleneck," i.e., a link $(i, j)$ where the minimum in (3) is attained. If there is only one such path, then the expression becomes exact. We thus propose to consider the ratio of the actual flow and the redundant capacity as a measure to predict critical links as

$$
\begin{aligned}
& \left|F_{a b} / K_{a b}^{\mathrm{red}}\right|>h \Rightarrow \text { predicted to be critical, } \\
& \left|F_{a b} / K_{a b}^{\mathrm{red}}\right| \leq h \Rightarrow \text { predicted to be stable, }
\end{aligned}
$$

where $h$ is a threshold value that can be optimized for any given specific prediction. Figure 2 illustrates that 

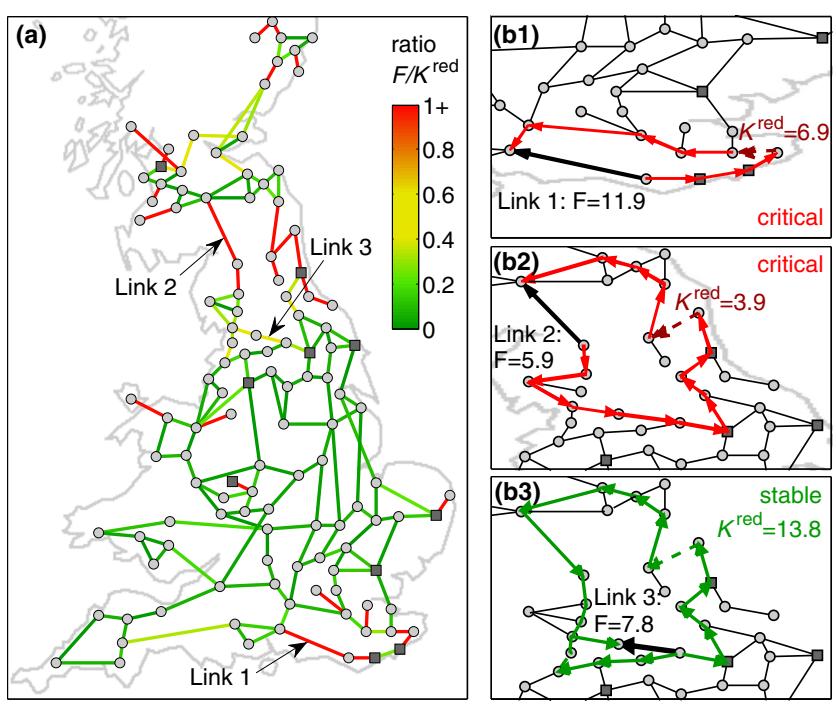

FIG. 2. Redundant capacity indicates flow rerouting options. (a) Ratio between flows and redundant capacities (color coded), with the three links from Fig. 1 indicated by arrows. (b) Dominant rerouting paths. (b1),(b2) For the two links (1, heavily loaded) and (2, moderately loaded), the flows to be rerouted are larger than the redundant capacity. The bottlenecks on the rerouting path are indicated by dashed red arrows. Thus, $\left|F_{a b} / K_{a b}^{\text {red }}\right|>1$ and these links are critical. (b3) For link 3, whereas more heavily loaded than link 2, the flow to be rerouted is smaller than the total available redundant capacity. Thus, $\left|F_{a b} / K_{a b}^{\mathrm{red}}\right|<1$ and the link is stable.

predictions based on redundant capacity (4) may work even for links where load-based predictions fail.

Flow rerouting in linear response.-Alternatively, we analyze how general alterations of the capacity of a single link modify the global operation of a network. Consider a small perturbation $\kappa_{i j}$ of the network capacities at a single link $(a, b)$ such that $K_{i j}^{\prime}=K_{i j}+\kappa_{i j}$ with $\kappa_{a b}=\kappa_{b a}=\kappa$ and $\kappa_{i j}=0$ for all other links. This perturbation induces a change $\phi_{j} \rightarrow \phi_{j}^{\prime}$ of the steady state phases of the network.

Expanding the perturbed steady state $\left[\ddot{\phi}_{j}^{\prime}=\dot{\phi}_{j}{ }^{\prime}=0\right.$ in Eq. (1)] to first order in the response $\xi_{j}:=\phi_{j}{ }^{\prime}-\phi_{j}$ yields

$$
\sum_{i} K_{i j} \cos \left(\phi_{i}-\phi_{j}\right)\left(\xi_{i}-\xi_{j}\right)=-\sum_{i} \kappa_{i j} \sin \left(\phi_{i}-\phi_{j}\right) .
$$

During normal operation, we can assume that $\left|\phi_{i}-\phi_{j}\right| \leq$ $\pi / 2$ for all links [59] and use (2) to obtain the identity $K_{i j} \cos \left(\phi_{i}-\phi_{j}\right)=\sqrt{K_{i j}^{2}-F_{i j}^{2}}=: \tilde{K}_{i j}$. This quantity characterizes the available capacity that the network may use to respond to perturbations in terms of the network topology and the original flows. We define the Laplacian matrix [62] $\Lambda_{i j}:=-\tilde{K}_{i j}+\delta_{i, j} \sum_{n} \tilde{K}_{i n}$, using the Kronecker symbol $\left(\delta_{x, y}=1\right.$ if $x=y$ and $\delta_{x, y}=0$ otherwise). With the vectorial components $q_{i}=\left(\delta_{i, a}-\delta_{i, b}\right)$, Eq. (5) reads $\Lambda \xi=$ $\kappa L_{a b} \boldsymbol{q}$. This equation is linear but depends nonlinearly on the system's unperturbed state variables $\phi_{i}$. Using the Moore-Penrose pseudoinverse $T:=\Lambda^{+}$yields the phase responses

$$
\xi=\kappa L_{a b} T \boldsymbol{q} .
$$

Thus, the power flow (2) in the perturbed network is to first order in $\kappa$ given by

$$
\begin{aligned}
F_{i j}^{\prime} & =\left(K_{i j}+\kappa_{i j}\right) \sin \left(\phi_{i}-\phi_{j}+\xi_{i}-\xi_{j}\right) \\
& =F_{i j}+\kappa \eta_{i j \leftarrow a b},
\end{aligned}
$$

where we have defined the link susceptibility $\eta_{i j \leftarrow a b}:=$ $L_{a b} \times\left[\tilde{K}_{i j}\left(T_{j b}-T_{j a}-T_{i b}+T_{i a}\right)+\left(\delta_{i a} \delta_{j b}-\delta_{j a} \delta_{i b}\right)\right]$. So if capacities are perturbed slightly, Eq. (7) tells us, to leading order in the perturbations $\kappa$, which flows increase, which decrease, and how much.

Even for perturbations $\kappa$ that are not small, this linear response argument reliably predicts where the flow is rerouted [31]. To predict the magnitude of the flow change also for a total link failure, we self-consistently renormalize the linear response by replacing $\kappa:=-F_{a b} / \eta_{a b \leftarrow a b}$, thereby ensuring that the flow of the defective link vanishes: $F_{a b}^{\prime}=0$. From this renormalized linear response theory, the modified estimates for the rerouted network flows read

$$
F_{i j}^{\prime \prime}=F_{i j}-\frac{\eta_{i j \leftarrow a b}}{\eta_{a b \leftarrow a b}} F_{a b} .
$$

The $F_{i, j}^{\prime \prime}$ do, of course, depend on the considered link $(a, b)$, that for clarity does not explicitly appear as an index. Related measures are used in engineering where they are referred to as line outage distribution factors [63].

As the rerouted total flows on each link must not be larger than the respective link capacities, we propose the predicted maximum load $\max _{(i, j)}\left|F_{i j}^{\prime \prime} / K_{i j}\right|$ as a discriminating feature between critical and stable links:

$$
\begin{aligned}
& \max _{(i, j)}\left|F_{i j}^{\prime \prime} / K_{i j}\right|>h \Rightarrow \text { predicted to be critical, } \\
& \max _{(i, j)}\left|F_{i j}^{\prime \prime} / K_{i j}\right| \leq h \Rightarrow \text { predicted to be stable. }
\end{aligned}
$$

In addition, every link whose breakdown disconnects the network is predicted to be critical.

This renormalized linear response theory not only well identifies which links are critical, based on criterion (9), it also predicts the value of the expected maximum load across the network [Fig. 3(a)].

Load and topology coact.-The response (6) and thus the susceptibility $\eta$ are directly proportional to the load $L_{a b}$ of a link. The load does determine its relevance, but other collective characteristics of the network are equally important.

For heavy loads, some of the responsive capacities $\tilde{K}_{i j}$ tend to zero and the associated network becomes weakly 

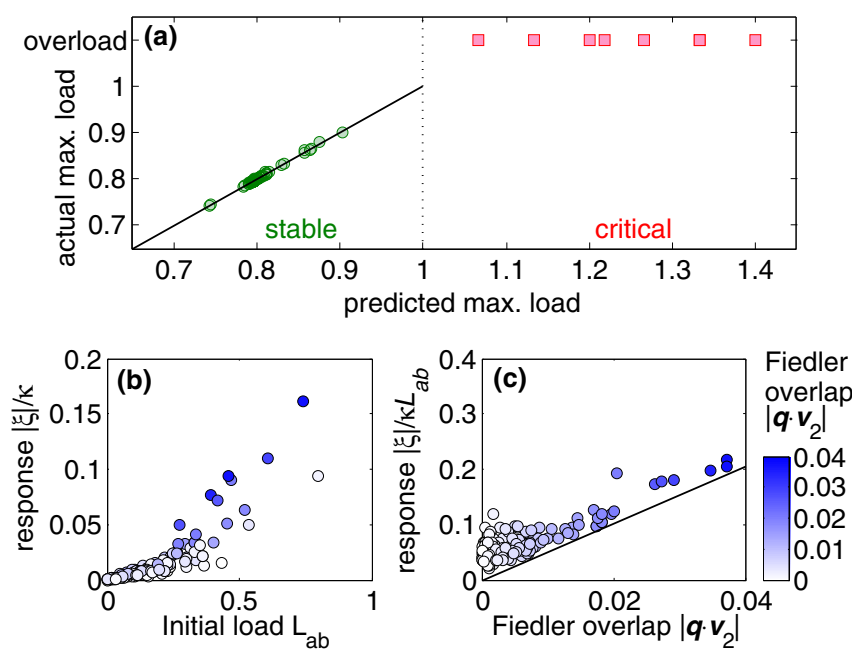

FIG. 3. The renormalized linear response theory predicts critical links. (a) The renormalized linear response not only predicts which links are critical (red squares) and which are stable (green disks), it also accurately tells the maximum loads for stable links (solid line, no fit parameter). (b) The response as a function of the load $L_{a b}$ of the failing link does grow proportionally to the load but also depends on other factors. (c) The response grows proportionally to the overlap $\left|\boldsymbol{q} \cdot \boldsymbol{v}_{2}\right|$ with the Fiedler vector. The solid straight line with slope $1 / \lambda_{2}$ gives a strict lower bound; see Eq. (10). Networks and parameters are as in Fig. 2.

connected. The second eigenvalue $\lambda_{2}$ of the Laplacian $\Lambda$, which measures the algebraic connectivity, becomes small (while $\lambda_{1} \equiv 0$, independent of the network and operating state) [62]. Then the pseudoinverse $T=\Lambda^{+}$is dominated by $1 / \lambda_{2}$, and the response is given by

$$
\boldsymbol{\xi} \approx \frac{\kappa L_{a b}}{\lambda_{2}}\left(\boldsymbol{v}_{2} \cdot \boldsymbol{q}\right) \boldsymbol{v}_{2},
$$

up to terms of the order of $1 / \lambda_{3}$. Here, $\boldsymbol{v}_{2}$ denotes the eigenvector associated with $\lambda_{2}$. Thus, the response is specifically also determined by two intrinsically collective properties of the load distribution: the inverse algebraic connectivity $1 / \lambda_{2}$ and the overlap $\left(\boldsymbol{v}_{2} \cdot \boldsymbol{q}\right)$, which is large for links connecting different components of the network $[31,64]$. Direct numerical simulations confirm these theoretical predictions [Figs. 3(b) and 3(c)].

Quality of critical link predictions. - The two proposed classifiers (4) and (9) enable the prediction of critical links with high accuracy. To test the performance, we generate 400 random network realizations, varying the generator positions, thereby analyzing 66000 links in total. A quantitative assessment of the different classifiers is provided by a receiver operating characteristic (ROC) curve (Fig. 4), displaying the fraction of correct predictions of critical links (the sensitivity) as a function of the fraction of false alarms (the false positive rate) when the discrimination threshold $h$ is varied. We see that the two classifiers introduced in this Letter closely approach the perfect operating point $(0,1)$ with almost no false alarms and

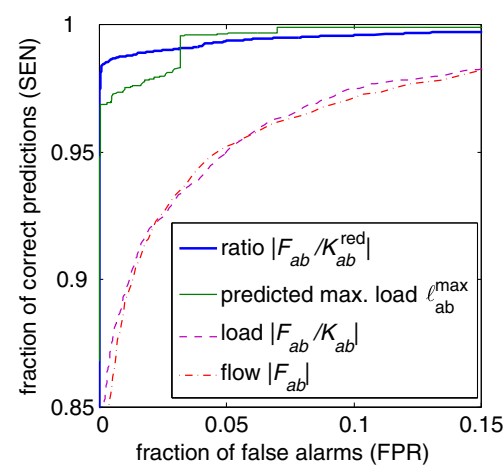

FIG. 4. Improved quality of the critical link prediction. The receiver operating characteristic curves indicate a multifold increase in the prediction quality using the classifiers (4) and (9) in comparison to the load or flow. (Statistics for 66000 links from 400 random grid realizations; model, topologies, and parameters are as in Fig. 1.)

almost perfect sensitivity (Fig. 4). The total number of incorrect predictions, including false alarms and missed critical links, is as small as $395(0.6 \%)$ for the ratio (4) and $920(1.4 \%)$ for the linear response measure (9). Thus, it is possible to reduce the incorrect predictions based on loads $(3149,4.7 \%)$ and flows $(2937,4.5 \%)$ by a factor of more than 7 , thereby drastically improving performance. Other macroscopic quantifiers such as those based on the area under the ROC curve confirm this view. A similar improvement is found for other test grids with different topologies and parameters [31].

The residual prediction errors indicate collective nonlinear effects in the grid. False alarms occur when the collective rerouting is significantly more efficient than assumed by the predictors. This occurs primarily when the flow change is strongly nonlinear, such that the linear response (9) is no longer appropriate or when the rerouted flow $\left|F_{a b}\right|$ in the redundant capacity measure (4) is large. In addition, this measure misses some critical links when there are several independent rerouting paths, as it assumes that all paths are used equivalently which is not always satisfied [31]. Hence, classifier (9) is preferable when $\left|F_{a b}\right|$ is large, while (4) is preferable when the load of all other edges is close to one.

Discussion.-In summary, we link the overall network topology with the load distribution resulting from the collective network dynamics and present nonlocal relations to identify a network's response to link failures. On this basis, we propose two network-based strategies to identify critical links by (i) quantifying the redundant capacity of the network and (ii) estimating the flow rerouting through developing a renormalized linear response theory. The analysis suggests that the two proposed predictors are well suited to identify critical links. In particular, they provide a substantial improvement of the quality of predicting critical links compared to predictions based on local measures such as a link's load. We emphasize that the quality of prediction 
is evaluated by statistical measures whereas the predicting measures are derived from insights about the topological connectivity and the nonlinear dynamics of the networks.

By construction, the proposed criteria readily generalize to other network topologies, to the physics of generic linear supply networks, and to complex load flow models as standard in engineering [31]. For network operation, the proposed predictors may provide key hints for initial analysis, because they suggest which links require special attention, e.g., in detailed large-scale simulations of power grid engineering. In particular, our insights about collective influences indicate that the weak spots of a network are not necessarily given by the most heavily loaded elements (cf. $[9,12]$ ) and that network-based rather than local measures provide suitable guidelines for the security assessment of real-world power grids (cf. [19]).

We thank Moritz Matthiae for valuable discussions and Peter Menck for providing network data. We gratefully acknowledge support from the Federal Ministry of Education and Research (BMBF Grants No. 03SF0472B and No. 03SF0472E to M. T. and D. W.), the Helmholtz Association (Grant No. VH-NG-1025 to D. W.), and the Max Planck Society to M. T.

[1] S. H. Strogatz, Nature (London) 410, 268 (2001).

[2] S. M. Amin and B. F. Wollenberg, IEEE Power Energy Mag. 3, 34 (2005).

[3] P. Pourbeik, P. Kundur, and C. Taylor, IEEE Power Energy Mag. 4, 22 (2006).

[4] E. Marris, Nature (London) 454, 570 (2008).

[5] W. Kröger, Reliab. Eng. Syst. Safe. 93, 1781 (2008).

[6] P. Hines, J. Apt, and S. Talukdar, Energy Policy 37, 5249 (2009).

[7] R. Albert, H. Jeong, and A. Barabási, Nature (London) 406, 378 (2000).

[8] R. Cohen, K. Erez, D. ben-Avraham, and S. Havlin, Phys. Rev. Lett. 86, 3682 (2001).

[9] R. Albert, I. Albert, and G. L. Nakarado, Phys. Rev. E 69, 025103 (2004).

[10] I. Simonsen, L. Buzna, K. Peters, S. Bornholdt, and D. Helbing, Phys. Rev. Lett. 100, 218701 (2008).

[11] S. V. Buldyrev, R. Parshani, G. Paul, H. E. Stanley, and S. Havlin, Nature (London) 464, 1025 (2010).

[12] P. Hines, E. Cotilla-Sanchez, and S. Blumsack, Chaos 20, 033122 (2010).

[13] D. Witthaut and M. Timme, New J. Phys. 14, 083036 (2012).

[14] D. Witthaut and M. Timme, Eur. Phys. J. B 86, 377 (2013).

[15] D. Helbing, Nature (London) 497, 51 (2013).

[16] D. Heide, L. von Bremen, M. Greiner, C. Hoffmann, M. Speckmann, and S. Bofinger, Renewable Energy 35, 2483 (2010).

[17] P. Milan, M. Wächter, and J. Peinke, Phys. Rev. Lett. 110, 138701 (2013).

[18] T. Pesch, H.-J. Allelein, and J.-F. Hake, Eur. Phys. J. Spec. Top. 223, 2561 (2014).
[19] R. Baldick et al., in Proceedings of the IEEE Power and Energy Society General Meeting-Conversion and Delivery of Electrical Energy in the 21st Century, 2008 (IEEE, New York, 2008), p. 1.

[20] E. Bompard, M. Masera, R. Napoli, and F. Xue, in Critical Information Infrastructure Security, Lect. Notes Comput. Sci. Vol. 5508, edited by R. Setola and S. Geretshuber (Springer, Berlin, 2009), p. 144.

[21] C. M. Schneider, A. A. Moreira, J. S. Andrade, S. Havlin, and H. J. Herrmann, Proc. Natl. Acad. Sci. U.S.A. 108, 3838 (2011).

[22] D. Grady, C. Thiemann, and D. Brockmann, Nat. Commun. 3, 864 (2012).

[23] P. J. Menck, J. Heitzig, J. Kurths, and H. J. Schellnhuber, Nat. Commun. 5, 3969 (2014).

[24] M. T. Schaub, J. Lehmann, S. N. Yaliraki, and M. Barahona, Network Sci. 2, 66 (2014).

[25] J. Salmeron, K. Wood, and R. Baldick, IEEE Trans. Power Syst. 19, 905 (2004).

[26] European Network of Transmission System Operators for Electricity, Continental Europe Operation Handbook, https://www.entsoe.eu/publications/system-operationsreports/operation-handbook.

[27] E. Katifori, G. J. Szöllősi, and M. O. Magnasco, Phys. Rev. Lett. 104, 048704 (2010).

[28] M. Rohden, A. Sorge, M. Timme, and D. Witthaut, Phys. Rev. Lett. 109, 064101 (2012).

[29] A. R. Bergen and D. J. Hill, IEEE Trans. Power Appar. Syst. PAS-100, 25 (1981).

[30] G. Filatrella, A. H. Nielsen, and N. F. Pedersen, Eur. Phys. J. B 61, 485 (2008).

[31] See Supplemental Material at http://link.aps.org/ supplemental/10.1103/PhysRevLett.116.138701 for more details on the power grid model, the calculation of the classifiers, the statistical assessment of classifiers, and additional data for other network topologies and other supply network models, which includes Refs. [32-50].

[32] J. J. Grainger and W. D. Stevenson, Jr., Power System Analysis (McGraw-Hill, New York, 1994).

[33] P. Kundur, Power System Stability and Control (McGraw-Hill, New York, 1994).

[34] J. Machowski, J. Bialek, and J. Bumby, Power System Dynamics, Stability and Control (Wiley, New York, 2008).

[35] F. Dörfler and F. Bullo, IEEE Trans. Circuits Syst. I 60, 150 (2013).

[36] Y. Kuramoto, in Proceedings of the International Symposium on on Mathematical Problems in Theoretical Physics, Lect. Notes Phys. Vol. 39, edited by H. Araki (Springer, New York, 1975), p. 420.

[37] S. H. Strogatz, Physica (Amsterdam) 143D, 1 (2000).

[38] J. A. Acebrón, L. L. Bonilla, C. J. Pérez Vicente, F. Ritort, and R. Spigler, Rev. Mod. Phys. 77, 137 (2005).

[39] K. Schmietendorf, J. Peinke, R. Friedrich, and O. Kamps, Eur. Phys. J. Spec. Top. 223, 2577 (2014).

[40] K. Purchala, L. Meeus, D. Van Dommelen, and R. Belmans, in Proceedings of the IEEE Power Engineering Society General Meeting (IEEE, New York, 2005), Vol. 1, pp. 454-459.

[41] D. Van Hertem, J. Verboomen, K. Purchala, R. Belmans, and W. Kling, in Proceedings of the 8th IEEE International 
Conference on ac and $d c$ Power Transmission (IEEE, New York, 2006), pp. 58-62.

[42] M. Durand and D. Weaire, Phys. Rev. E 70, 046125 (2004).

[43] R. D. Christie, Power systems test case archive, http://www .ee.washington.edu/research/pstca/.

[44] Union for the Coordination of Transmission of Electricity, Final report on the system disturbance on 4 November 2006, https://www.entsoe.eu/fileadmin/user_upload/_library/ publications/ce/otherreports/Final-Report-20070130.pdf.

[45] D. Jungnickel, Graphs, Networks and Algorithms (Springer, Berlin, 2012).

[46] M. Fiedler, Czech. Math. J. 23, 298 (1973).

[47] T. Güler and G. Gross, IEEE Trans. Power Syst. 22, 505 (2007).

[48] I. Rish, in Proceedings of the IJCAI 2001 Workshop on Empirical Methods in Artificial Intelligence (IBM, New York, 2001), pp. 41-46.

[49] H. Zhang, in Proceedings of the Seventeenth International Florida Artificial Intelligence Research Society Conference (Association for the Advancement of Artificial Intelligence, Palo Alto, 2004), p. 562.

[50] J. P. Egan, Signal Detection Theory and ROC Analysis (Academic, New York, 1975).

[51] A. Pikovsky, M. Rosenblum, and J. Kurths, Synchronization: A Universal Concept in Nonlinear Sciences (Cambridge University Press, Cambridge, England, 2003).
[52] P. Ashwin, O. Burylko, Y. Maistrenko, and O. Popovych, Phys. Rev. Lett. 96, 054102 (2006).

[53] M. Timme, Phys. Rev. Lett. 98, 224101 (2007).

[54] R. Zillmer, R. Livi, A. Politi, and A. Torcini, Phys. Rev. E 76, 046102 (2007).

[55] E. Schöll and H. G. Schuster, Handbook of Chaos Control (Wiley-VCH, Weinheim, 2008).

[56] M. Rohden, A. Sorge, D. Witthaut, and M. Timme, Chaos 24, 013123 (2014).

[57] F. Dörfler, M. Chertkov, and F. Bullo, Proc. Natl. Acad. Sci. U.S.A. 110, 2005 (2013).

[58] A. E. Motter, S. A. Myers, M. Anghel, and T. Nishikawa, Nat. Phys. 9, 191 (2013).

[59] D. Manik, D. Witthaut, B. Schäfer, M. Matthiae, A. Sorge, M. Rohden, E. Katifori, and M. Timme, Eur. Phys. J. Spec. Top. 223, 2527 (2014).

[60] R. K. Ahuja, T. L. Magnanti, and J. B. Orlin, Network Flows: Theory, Algorithms, and Applications (PrenticeHall, Englewood Cliffs, NJ, 1993).

[61] D. L. Alderson, G. G. Brown, M. W. Carlyle, and L. Anthony Cox, Mil. Oper. Res. 18, 21 (2013).

[62] M. E. J. Newman, Networks-An Introduction (Oxford University Press, Oxford, UK, 2010).

[63] A. J. Wood, B. F. Wollenberg, and G. B. Sheblé, Power Generation, Operation and Control (Wiley, New York, 2013).

[64] S. Fortunato, Phys. Rep. 486, 75 (2010). 